\title{
How MOOCs Are Perceived by Moroccan Students and Teachers?
}

\author{
Abdelaziz Razouki, Assistant Professor \\ Salah-eddine Khzami, Professor \\ Ikbal El Khatabi, PhD Student \\ Boujemaa Agorram, Professor \\ Sabah Selmaoui, Professor \\ Cadi Ayyad University, Marrakech, Morocco
}

doi: 10.19044/esj.2017.v13n22p82 URL:http://dx.doi.org/10.19044/esj.2017.v13n22p82

\begin{abstract}
The Moroccan university is increasingly experiencing a strong growth in the numbers of students. This process of massification alters the quality of learning, especially in open-access institutions where human resources and the capacity to host are increasingly limited. To improve the quality of learning, which first involves improving the quality of teaching within the University, Cadi Ayyad University in Marrakech (UCAM) launched a platform of MOOCs (Massive Online Open Courses) in 2013. In this article, we question the opening of the Moroccan university on this technology. Our study is anchored in the context of the Cadi Ayyad University of Marrakech. We are particularly interested in the following question: what are the perceptions of students and teachers about this device? Do they feel that using MOOCs "changes something"? Our methodological approach describes all the stages of data collection and processing including the content analysis model. We distributed a questionnaire to a group of students and conducted semi-structured interviews with teachers. The questionnaires collected and the interviews made it possible for answers to be provided to the questions asked. The most salient results show that the students as well as the teachers who participated in our study appreciate the use of MOOCs as a support to the face-to-face class. However, this technology has disadvantages including lack of exchanges with teachers and between students. Ongoing product improvements were called for, including the design in association with MOOC of student activities, assessments and discussion forums, and on the other hand, recording the videos in the presence of the students
\end{abstract}

Keywords: MOOCs, perceptions, students, teachers 


\section{Introduction}

Consequently, one can consider MOOCs (massive open online courses) to be an open, free, and online courses offered to a large number of learners (Karsenti et al., 2015). It is regarded as a new form of Teleeducation whose popularity in universities in USA, Europe, and other places can be described as a phenomenal in recent years. The MOOCs acronym was originally proposed in 2008 to designate a form of teaching that integrates the dimensions of the Web, according to a theory called "connectivism" (Siemens, 2005).

According to Ciseland and Bruillard (2012) and Grover et al. (2012), the emergence of the MOOCs reached its peak in 2012. However, its deployment in various forms is far from being complete (Daniel, 2012). This emergence deserves the attention of the academic community: decisionmakers, teachers, students, and researchers. Except if there is a great deal of scientific work on the relationship between teaching and digital devices, those devoted specifically to MOOC are very scarce. Nevertheless, this educational technology has attracted a great deal of practical interest. In fact, they are exponentially increasing, and their number has increased from 10 in 2011 to 4,000 in 2015 (Delpech \& Diagne, 2016). The fact is that despite its exponential development, some researchers still question the actual relevance of MOOCs. Are they more effective? Do they allow a better focus on teaching content?

According to Karsenti (2003), many studies have shown that a student can learn more and faster with ICTs. The authors of those researches also point out that Open and Distance learning is a "fascinating and unique" teaching and learning option. There are many advantages in terms of flexibility, accessibility, communication, increased interaction, and diversification of teaching and learning modes. According to an OECD report, training using MOOCs contributes significantly to the current development of higher education, although their progress is lower than projected. They can be described as "an approach that aims to broaden access to education and training services by enabling learners to overcome the barriers of space and time and by providing flexible learning opportunities for individuals and Groups of learners" (OECD, 2005).

Subsequently, their educational potential is of particular interest to a country such as Morocco. Here, universities are faced with strong growth in the numbers of students. The gradual integration of these technologies into the development of national curricula is an important step in the implementation of the education sector reform.

However, the Moroccan university is now facing several challenges and constraints that have a negative impact on higher education. These can be summarized into two major challenges: 
(1) The massification of the university, especially in the open-access institutions, which constitutes the majority of the students with a low pedagogical supervision in relation to the large number of students. This phenomenon has a detrimental effect on the quality of education.

(2) The high rate of failure of students, especially newcomers, and this goes from bad to worse every year. New entrants are the most affected by these problems. This makes it difficult to integrate them into higher education. Therefore, this occurs as a result of several factors, including:

- Difficulties linked to the passage from high school training system to the university system.

- The change in the language of instruction, which is Arabic in high school and French in university.

- $\quad$ The high number of learners in comparison with the human and material resources available to the university.

Furthermore, the university must now respond creatively and effectively to find solutions to the problems associated with the large flows of new entrants.

To meet these challenges, Cadi Ayyad University (UCA) has adopted a new strategy centered on the use of digital technology to fill or at least reduce the influence of problems that negatively affect student learning. The UC@MOOC project, which is the first experience in Morocco, was initiated by the UCA since 4 years ago. It is dedicated to the production of courses, tutorials, and practical work as a solution "that aims to give students the opportunity to access their teachers' courses and to learn anywhere and at any time and as many times as they desire it" (http://mooc.uca.ma/).

However, it is with this perspective that we approach the MOOC in our study. We recognized their potential in the development and improvement of the teaching-learning process in higher education. Also, we recognized the limits that should be met by using them within the Moroccan university.

Our study was carried out within Cadi Ayyad University which is the national leader in the field of MOOC and where an original experience in the development of online training for students is realized. Our research question is formulated as follows: What are the perceptions of students and teachers about MOOCs?

\section{Methodology}

In this study, we are interested in the perceptions that users and designers have on distance learning via MOOC. Our objectives are (1) to identify and describe the perceptions of both teachers and university students, (2) to compare teachers' perceptions, (3) to identify the difficulties 
encountered and elements of satisfaction with MOOCs training by determining the impact of this technology on the teaching-learning process.

We opted for a methodology based on a quantitative analysis of the data collected by means of a questionnaire. The questionnaire was distributed to a group of students and semi-structured interviews with teachers. Also, the questionnaire was developed on the basis of the review of the literature and the results of a pre-survey phase. After writing the final version of the questionnaire, it was distributed to a group of students from different fields (physics, biology, law, and economics) who received distance training via MOOCs. 340 students, of whom $45 \%$ were girls, completed the survey questionnaire.

The teacher sample consists of 9 out of the 45 UCA teachers involved in distance learning via MOOC, i.e. a participation rate of $20 \%$. The semidirected interviews of 14 questions lasted from 30 to 60 minutes and were recorded in mp3 format. During the interviews, the general questions were asked first before going on to the most precise questions. The answers to the questions in our interview protocol show a textual (faithful transcription of an oral statement) that we used to describe, analyze, and interpret the data collected.

The quantitative data collected were analyzed using the Sphinx lexica (V5) software. The analysis of the guided interviews was done by the coding of the open-ended questions. After that, the answers were analyzed in order to characterize the different types of answers.

\section{Results}

In this section, the results are presented in response to the research objective of identifying the perceptions of students and teachers on MOOCs.

\section{A. Perceptions of Students}

The analysis of the answers to the questions in the first part of the questionnaire shows that $89.4 \%$ of the students have access to a computer at home and $76.8 \%$ are connected to the Internet. This means that the majority have the necessary means to access the Distance training via MOOCs.

The other parts of the questionnaire gave results related to the advantages and disadvantages of MOOCs, rate of students' satisfaction, and impact of MOOCs on learning and teaching.

\section{The Advantages and Disadvantages of MOOCs}

The results show that in response to the question about what they see as benefits in MOOCs, $27 \%$ of students choose the response "the freedom to manage time", $26 \%$ choose "the opportunity to learn at one's own place" and $23 \%$ "a saving of time". 
Other students (16\%) think of "saving time and money" as a benefit related to the fact that the user does not have to travel to benefit from the MOOC technology. However, only a minority of students $(4 \%)$ noted that MOOCs can strengthen their learning capacity so that they can provide additional information and knowledge to students and also offer them the opportunity to better see and examine courses to deepen their learning.

The participants in our study therefore chose the benefits according to their needs. Thus, most of them attach great importance to the spatial and temporal dimensions and the pace of learning, which they consider to have an effect on learning.

Consequently, the analysis of the results also showed that a large proportion of the students questioned $(75.5 \%)$. This indicated that in the distance training via the MOOCs, "the lack of exchange with the teacher" represents the most felt disadvantage. Other students $(15 \%)$ choose "the fact that they have difficulty managing their time" as disadvantages of MOOCs use, while for others (3\%), the disadvantage is that "MOOCs has been a loss of time". The rest of the respondents had other disadvantages, such as "lack of supervision", "lack of assistance", and "the fact that MOOCs could lead students to believe that they can do without the face-to-face class".

The calculated difference between the results of the two sexes is not significant.

\section{MOOCs and the Face-to-Face Class}

The results showed that the majority of the students surveyed $(67 \%$ : $30.9 \%$ are girls and $36.2 \%$ are boys) said that MOOCs could not be an alternative to face-to-face class.

The small difference calculated between the two sexes $(5.3 \%)$ could be explained by the fact that distance education attracts more girls. This is because it is a more flexible characteristic in terms of time and place of study.

\section{Rate of Student Satisfaction}

The majority of respondents $(61.7 \%)$ appear not to be sufficiently satisfied at all by the quality of the MOOCs made available to them by their university (UCA). These students feel that this offer does not meet their expectations. Teachers should therefore be invited to enrich their MOOCs and develop them further.

\section{Appreciation and Usability of MOOCs}

The student's survey judged the MOOCs to be useful. The cumulative rates of the items "essential" and "useful" were $67.9 \%$ versus 
$32.1 \%$ for cumulative rates of the items "not very useful" and "not at all useful".

Moreover, the results also showed that the use of this device seems to be easy for the majority of the students (cumulative rates of the items "very easy" and "easy to use" is $76.5 \%$ against $23.5 \%$ cumulative rates of the items "difficult" and "very difficult"). That reflects the interest of young people, girls as well as boys in general for ICT and particularly MOOCs and their familiarity with those technologies.

This tells us that Moroccan students can learn with the MOOCs, especially since they have the necessary means (computers and Internet access) as mentioned above.

\section{Methods and Course Materials Used}

According to the results, we found that majority of the students affirm that the methods and the supports of online courses used by their teachers in the MOOCs are "unsuitable" to "very unsuitable".

\section{Impact on Ways of Teaching and Learning}

Only $38.8 \%$ of students say that MOOCs change the way teachers teach. The same proportion thinks the opposite, whereas a quarter of the sample has no opinion.

In response to the question "Does MOOCs change your way of learning?, it seems that most students $(75 \%)$ gave a positive answer. In addition, MOOC seems to have an influence on the learning process, at least according to the students' opinion.

\section{The Impact of MOOCs on Student Academic Outcomes}

MOOC appears to have a positive effect on the academic performance of more than half of the students surveyed (51\% chose the "agree" and "strongly agree" items).

\section{B. Perceptions of Teachers}

The analysis of the content of the interviews with nine teachers from various disciplines revealed four main lines of reflection: (1) the report of the teachers to the MOOCs, (2) the objectives and the performances carried out, (3) teaching by the MOOCs and in the classroom, (4) the interest and the future of the MOOCs at the university.

\section{Teachers' Report to MOOCs}

This first theme is related to three questions. The first relates to the reasons why the respondents proposed MOOCs. Thus, we want to know what motivated these teachers to get involved in this innovation. 
What motivates nearly half of the respondents seems to be first and foremost institutional in nature to join the university project to counter massification.

A third of the subjects explained that they chose to offer MOOCs mainly to facilitate access to information for students. Two teachers did so in order to provide another teaching medium that would complement the faceto-face class.

According to another teacher, the MOOCs seems to be a way to overcome the unfavorable conditions (overcrowding, bad sound, classroom disadvantage) in which classes take place.

Regarding the second question analyzed, "Today, are you satisfied with this choice because of the reasons that led you to teach by the MOOCs?", the respondents' speech testifies that the majority of them are satisfied with having proposed MOOCs. Nevertheless, they added that "there are many opportunities for improvement" or "yes, but I do not know what students think of these MOOCs". The other respondents say they cannot claim to be satisfied.

With regard to the third and last question of theme 1, "Is this experience positive?", the respondents' speech reflects similar views on the success of using MOOCs in university education. Thus, the experience is perceived positively by all the teachers interviewed.

\section{The Objectives and Performance Achieved}

In this section, we examine in greater detail the results obtained for the four questions related to theme 2. Also, we focus on the objectives of the MOOCs and on the performance achieved.

Regarding the first question, "What are the objectives of your university for the MOOCs?", the majority of teachers replied that the university aims above all that the MOOCs should play the role of support for the students.

Some argue that the only clear objective is "to reduce the pressure of large numbers on open access institutions" or "to limit mass flows at the class level". One teacher argues that the primary goal is to promote distance education.

Regarding the second question, "Does this tool help students to develop the skills targeted in the programs?", the majority of respondents think that the MOOCs does not allow the students to achieve this objective. Some argue that the only clear objective is "to reduce the pressure of large numbers on open access institutions" or "to limit mass flows at the class level".

One teacher believes in the possibility of skills development via MOOCs. However, he adds that resistance to any change in the system 
would hinder the achievement of such a goal. Others responded positively to the question, but they are not very sure.

The third question, "What is targeted learning and realized in MOOCs?", is addressed to teachers in order to know the type of learning targeted and the performances obtained. Only one of the teacher talked about the fact that in his MOOCs, he is targeting certain learning: "many types of learning including the development of autonomy, skills, written and oral communication, writing a synthesis, asking questions, comparing courses from different sources, translate, etc. ". Other respondents do not seem to differentiate between learning and understanding, or between learning and transmitting.

Regarding the fourth question: "What is the impact on students' performance?", half of the respondents talked about the increase in the success rate or the increase in the number of modules validated by the students. Others claim to be unable to confirm or deny that MOOCs have a positive impact on student performance.

\section{The Comparison of Teaching by MOOCs and Face-to-Face}

In this section, we want to examine the results obtained for the three issues related to theme 3 that deal with "teaching by MOOCs in relation to the face-to-face class". The answers of the teachers in regards to the first question, "Can the MOOCs replace the conferences?", show that they were unanimous in saying that the MOOCs were still teaching materials designed to help accompany the students and not to replace the lectures. Regarding the second question, "Do you think better to teach by MOOCs than in face-toface?", the majority of respondents leaned toward the face-to-face while some teachers did not see any difference between the two modes.

The analysis of the answers of the teachers to the third question, "Do you think that overall the student learns better by MOOCs than in the classroom?", shows that the opinions were divided.

\section{Interest and Future of MOOCs at the University}

This fourth theme is related to two questions: (1) what is the interest of MOOCs in your opinion? and (2) What do you think is the future of MOOC?

Some respondents to the first question believed that MOOCs are of particular interest in several ways: it is a tool that makes it possible to adapt education to real conditions by trying to circumvent constraints through the exploitation of great possibilities offered by modern media. Also, it creates an enormous dynamic of exchanges and skills development. Others claim to be unable to confirm or deny that MOOCs have a positive impact on student performance. The other teachers report that the MOOCs is certainly a very 
important means, but only helps to accompany the student. It helps him to follow and understand his course, set its own ideas, and improve his level.

One teacher talks about MOOCs as a learning medium, but he adds that it is necessary to look for ways to optimize its exploitation. Another suggested that MOOCs facilitates access to information, improves success, invites students to do research, and represents the brand image of the university and faculty.

\section{The Future of MOOCs}

At the end of these interviews, we asked the teachers about the future of MOOCs at the university. Teachers involved in MOOCs are largely convinced that this system will impose itself in a sustainable manner in the Moroccan educational landscape. The main reason is that it expands access to knowledge. Nearly half also think that MOOCs better respond to what learners expect from distance teaching. In addition, this is an innovation that will be irreversible. However, they demand that MOOCs should be developed and improved.

According to one teacher, the future is certain, provided that more teachers adhere to the project. Another insists that MOOCs must be designed that take our context into account and not just emulate MOOCs that has been designed elsewhere.

\section{Discussion}

It is clear from the results that, according to the students, despite the advantages of the MOOCs and its role in higher education, it cannot replace the normal course or the face-to-face class. The latter is always necessary and the students give great importance to the interactions with the teachers. In all of the proposed MOOCs, there is a lack of exchange with teachers. Also, there is an inability to directly ask in-depth questions to teachers. One also regrets the lack of interactions between students. This is probably the reason why the majority of students are not satisfied with this technology.

The results of this study also show that there is a relatively positive effect of MOOCs on student achievement. More than half of the students surveyed confirmed it and also claimed that there is an improvement in their learning as well. This result resembles that demonstrated in Rogers study (2004). Furthermore, the university platform gives students the opportunity to examine their courses at home in more appropriate conditions than in an overcrowded amphitheater where acquiring knowledge is difficult.

However, the results show a difference in perception among students and teachers about the impact of MOOCs on academic success. Teachers show more reserve, caution, and realism. While they do not deny the 
MOOCs' cognitive potential, they stressed that they do not systematically produce a positive effect on academic achievement.

On the other hand, despite the students' positive perception of the benefits of MOOCs integration in order to improve their learning and to cope with the difficulties and problems encountered, they argue that MOOCs is just a complement to the face-to-face class. It is therefore necessary to improve and further develop the MOOCs by trying to integrate the discussion forums with the teachers. It is also done between the students and the exercises accompanied by their answers to meet the needs of the students.

As demonstrated by the description and analysis of the data collected, the designers of the courses put on-line have in common the fact of having chosen to propose MOOCs in response to the request of the presidency of the university. The objective is to "find solutions to the problems associated with the large flows of new students while contributing to the improvement of the quality of learning and the training provided".

It is clear from the results obtained that the expectations of teachers are relatively clear and coherent. Subsequently, the emphasis is put on cognitive (especially conceptual) aspects and not on the learning process (the tasks to be performed and their sequence). Teachers focus on compelling the student to follow the proposed MOOCs. The offer is therefore partial.

Moreover, teachers appear not to be very productive in terms of pedagogical innovation and the production of digital content. Students consider what is offered just as a complement that they can consult before or after the in-class course to properly assimilate the taught contents and improve their learning. The results also indicate that the majority of teachers adopt a transmissive pattern in continuity with current practices. Apparently, they conceive the platform as an extension of the class in terms of space and time. Therefore, the same choices governing the traditional course came back in online courses.

This under-utilization of the MOOCs can be explained by several reasons, among which include: the absence of texts regulating distance diplomas, the lack of availability and involvement of teachers, and the lack of skills required for on-line course design.

The teachers interviewed indicated that the proposed MOOCs could not replace face-to-face training. This leads us to reflect on the future of face-to-face training and to develop further in the years to come the "hybrid approach" that would make it possible to reconcile between face-to-face class and e-Learning.

Despite teachers' positive perceptions of the integration of MOOCs, we find that they are content with minimal use of this technology. Also, they 
are difficulty which challenges their current pedagogical practices in favor of other more innovative technology.

The majority of teachers emphasize the fact that putting online courses requires teachers to invest their time and effort heavily. Therefore, everyone agrees that this investment gives rise to great satisfaction.

The teachers interviewed are mainly convinced that the MOOCs will impose themselves in a sustainable way in the Moroccan educational landscape. The main reason is that MOOCs expand access to knowledge. Nearly half of the teachers also think that they better respond to what learners expect from distance learning and that this is an innovation that will be irreversible. Those teachers want the MOOC's pedagogical model to be the result of local reflection.

\section{Conclusion}

In sum, the results of the study show that university students opt to turn to distance education because this technology offers much more flexibility than face-to-face training (non-compulsory presence, resolution of the geographical remoteness issue etc.). However, this type of training is not a system with only advantages. It also has disadvantages including the lack of exchanges with teachers and with other students. According to students as well as teachers, interaction is an important dimension that should be maintained even in virtual teaching.

Despite their affirmation of the positive impact of the MOOCs on the teaching-learning process, both populations draw attention to the need for further improvement of the MOOCs produced at the UCA until now. Students are requesting activities, assessments, and discussion forums in parallel with MOOCs. Teachers on their part want the videos of the classes to be recorded in the presence of the students.

Therefore, this study did not take into account the differentiation of students according to their academic levels. An extension of this survey to the various academic institutions should make it possible to identify students' specific needs in this field in order to develop adapted MOOCs. Studies should be carried out so as to analyze the factors explaining the students' motivation (academic experiences, ability to learn, socioeconomic factors, etc.) in order to better etch their needs.

\section{References:}

1. Daniel, J. (2012). Making Sense of MOOCs: Musings in a Maze of Myth, Paradox and Possibility, in Journal of Interactive Media in Education (JIME). Retrieved from: http://jime.open.ac.uk/articles/10.5334/2012-18/.

2. Grover, S., Franz, P., Schneider, E. \& Pea, R. (2012). The MOOC as 
Distributed Intelligence : Dimensions of a Framework for the Design and Evaluation of MOOCs. In Proceedings of the $10^{\text {th }}$ International Conference on Computer Supported Collaborative Learning, Madison, WI. http://mooc.uca.ma/.

3. Karsenti, T., Depover, C., Komis, V. \& Dumouchel, G. (2015). La pédagogie universitaire face aux MOOCs : compte-rendu d'expériences, tendances actuelles et futures, International Journal of Technologies in Higher Education, 12 (1-2), 7-8.

4. Karsenti, Th. (2003). Conditions d'efficacités des formations ouvertes ou à distance en pédagogie universitaire. Revue internationale francophone d'éducation médicale 4(4), 223-233. Retrieved from: https://www.pedagogiemedicale.org/articles/pmed/pdf/2003/04/pmed20034p223.pdf.

5. Matthieu Cisel \& Bruillard Éric (2012). Chronique des MOOC, Sciences et Technologies de 1'Information et de la Communication pour l'Éducation et la Formation, Volume 19, ISSN : 1764-7223. Retrieved from:

6. http://sticef.univ-lemans.fr/num/vol2012/13rcisel/sticef_2012 cisel_13r.htm

7. OCDE (2005). La cyberformation dans l'enseignement supérieur : état des lieux - ISBN 92-64-00922-1 (C) OCDE 2005. Retrieved from : http://www.oecd.org/fr/innovation/recherche/35028671.pdf.

8. Quentin \& Diagne Marième (2016). MOOC, l'âge de maturité ? Modèles économiques et évolutions pédagogiques, Retrieved from: http://www.strategie.gouv.fr/sites/strategie.gouv.fr/files/atoms/files/dt mooc_03022016_final.pdf.

9. Rogers, G. (2004). History, learning technology and student achievement: Making the difference?, Active Learning in Higher Education, 5(3), 232-247. Retrieved from http://journals.sagepub.com/doi/pdf/10.1177/146978740404381.1

10. Siemens, G. (2005, January). Connectivism: A learning theory for the digital age. International Journal of Instructional Technology \& Distance Learning. Retrieved from http://www.itdl.org/Journal/Jan_05/article01.htmDelpech. 